\title{
High-Temperature Adiabatic Calorimeter for Constant-Volume Heat Capacity Measurements of Compressed Gases and Liquids
}

\author{
Joseph W. Magee ${ }^{1}$ \\ National Institute of Standards and \\ Technology, \\ Boulder, CO 80303 \\ and \\ Renee J. Deal and John C. Blanco \\ University of Colorado, \\ Boulder, CO 80303
}

\author{
A high-temperature adiabatic calorimeter \\ has been developed to measure the con- \\ stant-volume specific heat capacities $\left(c_{V}\right)$ of \\ both gases and liquids, especially fluids \\ of interest to emerging energy technologies. \\ The chief design feature is its nearly \\ identical twin bomb arrangement, which al- \\ lows accurate measurement of energy \\ differences without large corrections for en- \\ ergy losses due to thermal radiation \\ fluxes. Operating conditions for the \\ calorimeter cover a range of temperatures \\ from $250 \mathrm{~K}$ to $700 \mathrm{~K}$ and at pressures up \\ to $20 \mathrm{MPa}$. Performance tests were made
}

with a sample of twice-distilled water. Heat capacities for water were measured from $300 \mathrm{~K}$ to $420 \mathrm{~K}$ at pressures to $20 \mathrm{MPa}$. The measured heat capacities differed from those calculated with an independently developed standard reference formulation with a root-mean-square fractional deviation of $0.48 \%$.

Key words: adiabatic; calorimeter; gases; heat capacity; isochoric; liquids; measurements; water.

Accepted: September 26, 1997

\section{Introduction}

Reliable thermal property data are required for efficient design in chemical engineering, as well as the enhancement of scientific understanding. The energy needed to increase the temperature of a kilogram of substance by $1 \mathrm{~K}$ (the specific heat capacity) is a quantity of considerable interest for many industrial applications. It also represents a fundamental measure of energy storage in the translational, rotational, and vibrational modes of a molecule and, as such, is useful in molecular theories.

An extensive array of techniques [1] have been developed to measure heat capacity. Of these techniques, the adiabatic method is generally accepted to give the most accurate results. The adiabatic method has been employed for heat capacity measurements by our research group for more than 35 years. Goodwin [2] developed a low-temperature adiabatic calorimeter, which was later

\footnotetext{
${ }^{1}$ Corresponding author.
}

modified by Magee [3] for automated measurement and control. It has been used to measure constant-volume heat capacities for many fluids at temperatures from $20 \mathrm{~K}$ to $345 \mathrm{~K}$, and at pressures to $35 \mathrm{MPa}$. In the planning stages of this work, we recognized a need to augment our existing measurement capabilities by developing a new calorimeter which would extend the upper temperature limit to $700 \mathrm{~K}$, without a compromise in accuracy.

In the adiabatic method, heat exchange between the calorimeter and its environment is eliminated as long as a temperature gradient does not exist. However, in actual practice the maintenance of a zero temperature gradient is an ideal situation which cannot be realized. Thus, even the most carefully conducted calorimetric experiment results in some heat loss. This heat loss can be minimized by automatic adjustment of the temperature of the surrounding jacket to follow that of the calorimeter. 
Since in practice we are only able to minimize heat losses, the primary problem becomes how to accurately evaluate the correction for heat leakage. Fortunately, the magnitude of the heat-loss term is much less important than the accuracy with which it can be determined. Since an accurate calculation of heat leakage is difficult and often not possible, it would be advantageous to develop a technique which eliminates the need to make such a correction altogether. Early attempts to accomplish this goal employed twin calorimeters. Twin calorimeters were used by Joule [4] in the mid-nineteenth century and later by Pfaundler [5]. Such twin devices have in common two calorimeters as nearly identical in construction as possible, supported in nearly identical surroundings. In the present work, we combine the features of twin calorimeters with the adiabatic method, with the goal of realizing virtually complete elimination of the heat-loss correction.

\section{Experimental Apparatus}

Almost identical spherical bombs of $70 \mathrm{~cm}^{3}$ capacity were designed to have a burst pressure in excess of 100 $\mathrm{MPa}$ at $700 \mathrm{~K}$. The bombs were fabricated from Inconel 718 because of its high strength and excellent corrosion resistance. It is a weldable, machinable nickel-chromium alloy having exceptionally high tensile strength at elevated temperatures. Its tensile strength is more than twice that of Type-316 stainless steel at $700 \mathrm{~K}$. A pair of these bombs was made from Inconel 718 steel sheet by spinning a hardened steel tool in a lathe to produce hemispheres which were subsequently welded together. As shown in Fig. 1, each hemisphere was machined with a face which is flat within close tolerances, and has a $60^{\circ}$ beveled edge to permit better access by the welding tool when two halves are fused together. After the hemispheres were fused, a $0.16 \mathrm{~cm}$ diameter filler rod of the same composition was melted into the groove. After the excess filler material was removed by grinding, the surface was polished to give a smooth appearance to the sphere. A $0.64 \mathrm{~cm}$ OD Inconel 718 tube was then welded into a hole of the same size in the top of the sphere. The assembly was then annealed while it underwent an argon purge at $1200 \mathrm{~K}$ to remove any heat-induced stress. Each of the spheres was then radiographed to reveal flaws in the weldments, if any. Radiographs of the spheres were made at a wide angle to the weldment. This angled view would reveal any areas which were only partially fused together. Such flaws would have appeared as darkened areas. No flaws were detected. When it was established that the metal was fused through the entire wall, the cells were tested under high vacuum for any leaks. Then they were hydrostatically pressure-tested to 1.75 times the maximum operating pressure (to $35 \mathrm{MPa}$ ) to establish their strength. Wall stress calculations indicate that the upper pressure limit at $700 \mathrm{~K}$, when the yield strength is exceeded, is approximately $110 \mathrm{MPa}$.

Selecting the heater wire and attaching this wire to the spheres proved to be most vexing problems. Most high-temperature wire insulations, such as polyimide and polytetrafluoroethylene, break down at temperatures greater than about $500 \mathrm{~K}$. Epoxy adhesives also degrade at similar temperatures. An objection to silicone rubber is its propensity to outgas in vacuum. The choice for this work was a metal-sheathed heater which could be brazed onto the surface. An Inconel-sheathed,

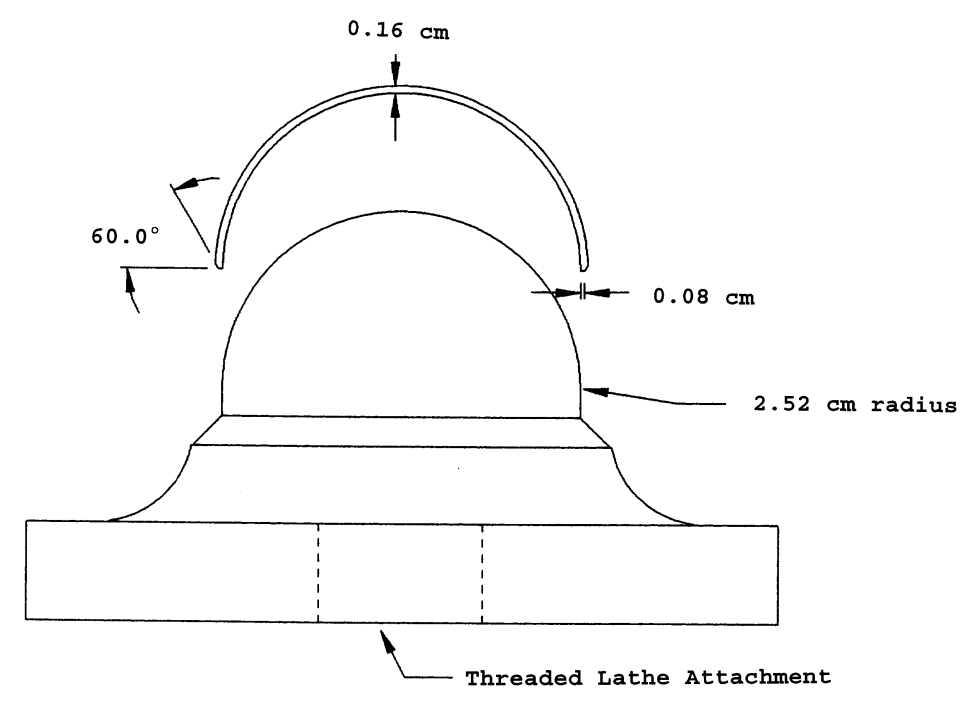

Fig. 1. Details of Inconel hemispheres. 
magnesium oxide insulated, nickel-chromium heater was brazed to the surface with a hard silver alloy that melted at about $900 \mathrm{~K}$. A light steel jig was designed and built to hold the wires in place during brazing.

As shown in Fig. 2, a light copper case was brazed to the sphere, covering two-thirds of its surface. It has two purposes. Chiefly, it intercepts stray heater radiation and conducts it back to the surface, thereby eliminating heat losses. Also, it serves as an anchor for the thermocouple well and platinum resistance thermometer (PRT) sheath which are brazed to it.

A capillary was attached to each sphere to fill and empty the vessels from outside the heated zone of the apparatus. A $0.05 \mathrm{~cm}$ inner diameter capillary was welded into a plug, which was in turn welded to the top of each $0.64 \mathrm{~cm}$ OD tube. Silver-alloy brazing could not be used to bond the parts of each sphere because of a known tendency of silver to catalyze decomposition of some hydrochlorofluorocarbon sample fluids at temperatures above $400 \mathrm{~K}$. Silver and other brazing alloy components, notably copper, are also susceptible to corrosion with substances such as ammonia. The integrity of the weld seals was verified with a helium leak detector.
Each completed spherical bomb was jacketed in an adiabatic shield coupled to a guard ring, all constructed of Type-6061 aluminum, which was selected for its machinability and high thermal conductivity. The cylindrical surfaces of the guard ring and the sides and bottom of the shield were machined with spiral grooves to accept heaters which were pressed into them. The entire calorimeter assembly, shown in Fig. 2, has been placed inside a high-temperature forced-air convection furnace, specially designed for this application. A differential thermocouple provides a continuous reading of the temperature difference between the sample and the reference cells. A three-junction differential thermopile provides readings of the temperature difference between each bomb and its associated adiabatic shield. Type-K thermocouples encased in magnesium oxide insulation and sealed in a steel sheath were selected for this duty. The measuring ends of the three thermocouples were brazed to the inside surface of the shield with the active portion inserted in three holes which were separated by a $120^{\circ}$ angle from each other and located at an elevation near the center of the bomb. The three reference ends were bound together and placed in the thermocouple

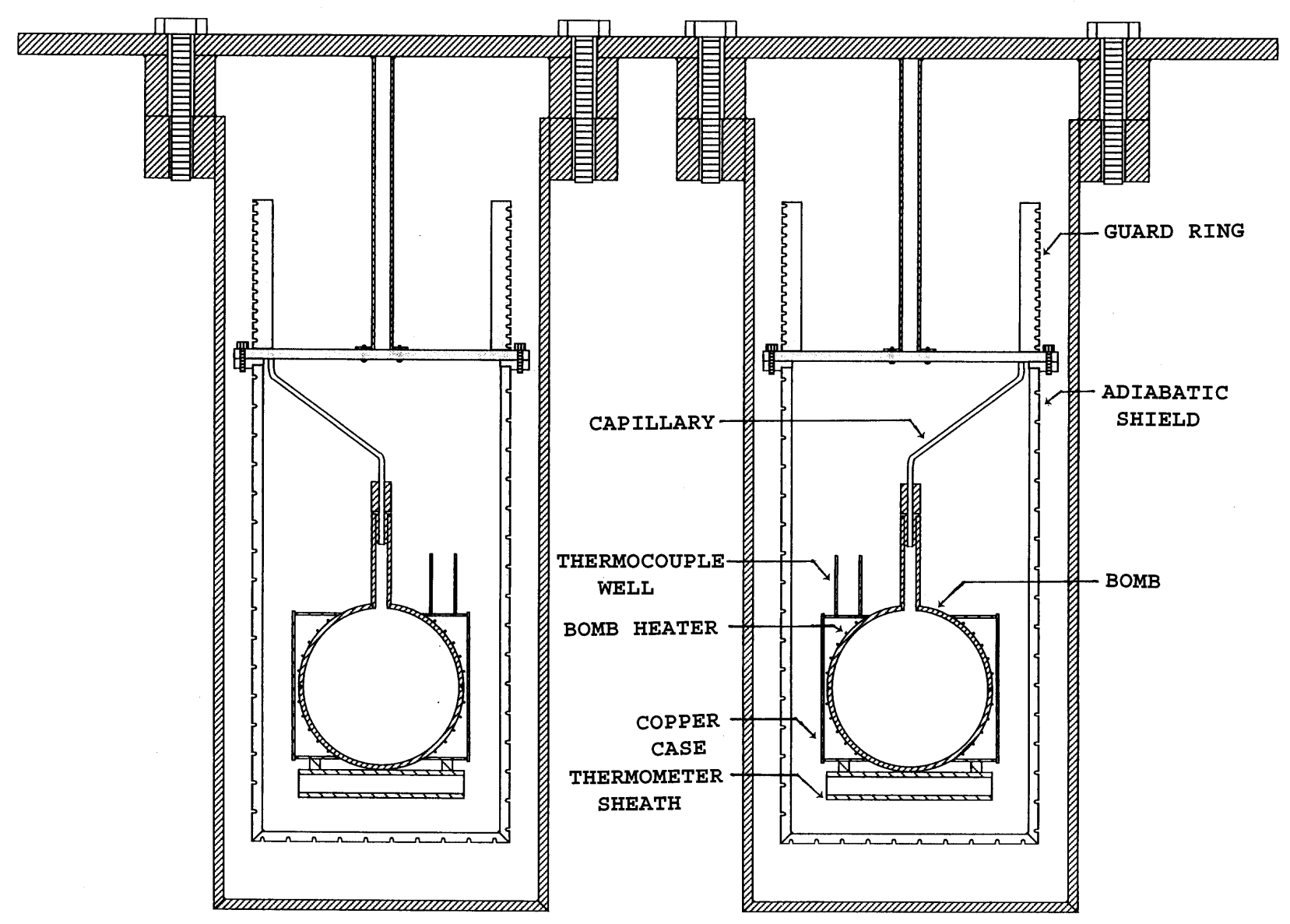

Fig. 2. Schematic drawing of twin calorimeter. 
well which contained thermally conductive grease to a depth of $1 \mathrm{~cm}$. A differential Type-K thermocouple provides readings of the temperature difference between the guard ring and the bomb. It was attached at each end in the same way as the shield thermocouples. A platinum resistance thermometer (PRT), having a calibration traceable to the National Institute of Standards and Technology, was coated with thermal grease and then inserted into the well brazed to the bottom of each bomb. These two thermometers provide measurements of the absolute temperature of each sphere with a high precision and accuracy. An oscillating quartz crystal pressure transducer, attached to the charging manifold, provides measurements of pressure. These pressures are nearly as accurate as the NIST-traceable piston gauges used to calibrate them. Each of the eight heaters (bomb, shield side, shield bottom, and guard for each cell) in the calorimeter is driven by independently controlled direct current power supplies. All of the instrumentation is connected to a microcomputer through an IEEE-488 standard interface bus. The computer executes FORTRAN code which has both temperature control and data acquisition functions. Except for sample charging, the new calorimeter is completely automated.

\section{Principle of Operation}

The basic principles of the heat capacity experiment are deceptively simple. For a single bomb, we measure the temperature rise $(\Delta T)$ when a measured quantity of heat energy $(Q)$ is supplied to the calorimeter, which contains a mass $(m)$ of substance. Subtracting the heat energy needed to heat the empty bomb $\left(Q_{0}\right)$ also widely known as heat equivalent, we calculate the sample heat capacity,

$$
c_{V}=\left(Q-Q_{0}\right) / m \Delta T .
$$

For a twin calorimeter, we simply replace values for $Q$ with the differential quantity, $\Delta Q$. It is defined as the heat energy supplied to the sample bomb minus that to the reference. The value of $\Delta Q$ accounts for the heat absorbed by the sample fluid. When we subtract the energy difference between the empty sample bomb and the reference, $\Delta Q_{0}$, we calculate the specific heat capacity at constant volume,

$$
c_{V}=\left(\Delta Q-\Delta Q_{0}\right) / m \Delta T .
$$

Equation (2) is the apparatus working equation. Experience with a single-cell calorimeter, has shown that the quantity $Q_{0}$ in Eq. (1) is as much as $95 \%$ of the measured heat energy $Q$. As a result, the relative expanded uncertainty (coverage factor $k=2$ and thus a 2-standarddeviation estimate) propagated to $c_{V}$ from the total uncertainty in evaluating $Q_{0}(\approx 0.02 \%)$ may be up to $0.4 \%$. This shortcoming of the single-bomb calorimeter leads to an estimated expanded uncertainty for the heat capacity of $0.5 \%$ for liquid and $2 \%$ for gas samples [3]. A twin-bomb calorimeter overcomes this problem. The quantity $\Delta Q_{0}$ in Eq. (2) is small by design and, in practice, is nearly 0 . The result of this technique is to make the uncertainty propagated from the heat equivalent measurements a negligible effect. Thus, we have improved the accuracy of the measurements, allowing us to achieve our goal of a relative expanded uncertainty of $0.4 \%$ for the measured specific heat capacities at constant volume.

\section{Performance Tests}

The empty calorimeter function was determined from heating the completely evacuated bombs. Heating runs over the temperature range $300 \mathrm{~K}$ to $400 \mathrm{~K}$ were repeated until we were confident in the precision of the results. The data were fit by the function,

$$
\Delta Q_{0}=2.13785 \times 10^{-2} \mathrm{~J} \cdot \mathrm{K}^{-1} \mathrm{~T}-5.86322 \mathrm{~J} .
$$

Over 300 individual measurements agreed with Eq. (3) within a maximum deviation of $\pm 0.5 \mathrm{~J}$. This equation is linear in temperature and ranges from $0.5 \mathrm{~J}$ to $3 \mathrm{~J}$ at temperatures from $300 \mathrm{~K}$ to $400 \mathrm{~K}$.

A twice-distilled sample of water was prepared for a performance test of the new calorimeter. The water was charged into the calorimeter with a high-pressure syringe pump. Excess sample was slowly removed until the target pressure was reached. Measurements were initiated by applying a constant $3 \mathrm{~V}$ to the reference bomb heater. The low power $(0.075 \mathrm{~W})$ dissipated in this $120 \Omega$ heater resulted in a temperature ramp rate of 0.04 $\mathrm{K} \cdot \mathrm{min}^{-1}$. The computer code quickly adjusted the sample bomb voltage to keep the bombs in thermal equilibrium within $\pm 5 \times 10^{-3} \mathrm{~K}$. When at equilibrium, the computer recorded the raw data needed for the heat capacity calculations. Heating cycles continued until the fluid pressure reached $20 \mathrm{MPa}$, the maximum pressure. Then the instruments were automatically reset to cool to the initial temperature of the run, and the run was repeated. After measurements were completed for a given isochore, a small amount of fluid was discharged into a light stainless-steel cylinder for weighing. After the last run, the remaining sample fluid was weighed.

Table 1 presents measurements of specific heat capacity at constant volume for water. The measurements are depicted in Fig. 3. To facilitate the comparisons with 
Table 1. Measurements of specific heat capacity at constant volume $c_{V}$ for $\mathrm{H}_{2} \mathrm{O}: T$, temperature (ITS-90); $p$, pressure; $m$, sample mass; $c_{V, \text { exp }}$, experimental heat capacity; $c_{V \text {,calc }}$, heat capacity calculated with the equation of state of Ref. [7]; $D=100$ $\left(c_{V, \exp }-c_{V, \text { calc }}\right) / c_{V, \exp }$

\begin{tabular}{|c|c|c|c|c|c|c|c|c|}
\hline $\begin{array}{r}T_{1} \\
(\mathrm{~K})\end{array}$ & $\begin{array}{r}T_{2} \\
(\mathrm{~K})\end{array}$ & $\begin{array}{c}p_{1} \\
(\mathrm{MPa})\end{array}$ & $\begin{array}{c}p_{2} \\
(\mathrm{MPa})\end{array}$ & $\begin{array}{c}\Delta p \\
(\mathrm{MPa})\end{array}$ & $\begin{array}{l}m \\
(\mathrm{~g})\end{array}$ & \multicolumn{2}{|c|}{$\begin{array}{c}c_{V, \exp } \quad c_{V, \text { calc }} \\
\left(\mathrm{kJ} \cdot \mathrm{kg}^{-1} \cdot \mathrm{K}^{-1}\right)\end{array}$} & $\begin{array}{c}D \\
(\%)\end{array}$ \\
\hline 304.0 & 305.0 & 1.0100 & 1.4560 & 0.4460 & 69.3106 & 4.1288 & 4.1052 & 0.571 \\
\hline 305.0 & 306.0 & 1.4560 & 1.9460 & 0.4900 & 69.3106 & 4.1232 & 4.0995 & 0.575 \\
\hline 306.0 & 307.0 & 1.9460 & 2.4670 & 0.5210 & 69.3106 & 4.1115 & 4.0936 & 0.434 \\
\hline 307.0 & 308.0 & 2.4670 & 3.0140 & 0.5470 & 69.3106 & 4.1233 & 4.0877 & 0.863 \\
\hline 308.0 & 309.0 & 3.0140 & 3.5830 & 0.5690 & 69.3106 & 4.1099 & 4.0818 & 0.685 \\
\hline 309.0 & 310.0 & 3.5830 & 4.1680 & 0.5850 & 69.3106 & 4.0939 & 4.0757 & 0.443 \\
\hline 310.0 & 311.0 & 4.1680 & 4.7870 & 0.6190 & 69.3106 & 4.0889 & 4.0696 & 0.471 \\
\hline 311.0 & 312.0 & 4.7870 & 5.4360 & 0.6490 & 69.3106 & 4.0695 & 4.0634 & 0.149 \\
\hline 312.0 & 313.0 & 5.4360 & 6.0540 & 0.6180 & 69.3105 & 4.0356 & 4.0573 & -0.539 \\
\hline 313.0 & 314.0 & 6.0540 & 6.7230 & 0.6690 & 69.3105 & 4.0555 & 4.0511 & 0.108 \\
\hline 314.0 & 315.0 & 6.7230 & 7.3860 & 0.6630 & 69.3105 & 4.0310 & 4.0449 & -0.345 \\
\hline 315.0 & 316.0 & 7.3860 & 8.0820 & 0.6960 & 69.3105 & 4.0510 & 4.0386 & 0.306 \\
\hline 316.0 & 317.0 & 8.0820 & 8.7860 & 0.7040 & 69.3105 & 4.0514 & 4.0323 & 0.471 \\
\hline 317.0 & 318.0 & 8.7860 & 9.5050 & 0.7190 & 69.3105 & 4.0350 & 4.0260 & 0.224 \\
\hline 318.0 & 319.0 & 9.5050 & 10.2170 & 0.7120 & 69.3105 & 4.0211 & 4.0197 & 0.035 \\
\hline 319.0 & 320.0 & 10.2170 & 10.9690 & 0.7520 & 69.3105 & 4.0172 & 4.0133 & 0.097 \\
\hline 320.0 & 321.0 & 10.9690 & 11.7320 & 0.7630 & 69.3105 & 4.0281 & 4.0069 & 0.527 \\
\hline 321.0 & 322.0 & 11.7320 & 12.5010 & 0.7690 & 69.3104 & 4.0102 & 4.0005 & 0.242 \\
\hline 322.0 & 323.0 & 12.5010 & 13.2870 & 0.7860 & 69.3104 & 4.0178 & 3.9941 & 0.591 \\
\hline 324.0 & 325.0 & 14.0830 & 14.8910 & 0.8080 & 69.3104 & 3.9919 & 3.9812 & 0.269 \\
\hline 325.0 & 326.0 & 14.8910 & 15.7160 & 0.8250 & 69.3104 & 3.9934 & 3.9747 & 0.468 \\
\hline 326.0 & 327.0 & 15.7160 & 16.5570 & 0.8410 & 69.3104 & 3.9998 & 3.9682 & 0.790 \\
\hline 327.0 & 328.0 & 16.5570 & 17.4060 & 0.8490 & 69.3104 & 3.9619 & 3.9617 & 0.004 \\
\hline 328.0 & 329.0 & 17.4060 & 18.2650 & 0.8590 & 69.3104 & 3.9628 & 3.9552 & 0.191 \\
\hline 304.0 & 305.0 & 1.0030 & 1.4490 & 0.4460 & 69.3106 & 4.1239 & 4.1052 & 0.453 \\
\hline 305.0 & 306.0 & 1.4490 & 1.9390 & 0.4900 & 69.3106 & 4.1082 & 4.0995 & 0.212 \\
\hline 306.0 & 307.0 & 1.9390 & 2.4590 & 0.5200 & 69.3106 & 4.1210 & 4.0937 & 0.663 \\
\hline 307.0 & 308.0 & 2.4590 & 3.0050 & 0.5460 & 69.3106 & 4.1041 & 4.0878 & 0.398 \\
\hline 309.0 & 310.0 & 3.5720 & 4.1620 & 0.5900 & 69.3106 & 4.0612 & 4.0758 & -0.359 \\
\hline 310.0 & 311.0 & 4.1620 & 4.7780 & 0.6160 & 69.3106 & 4.0717 & 4.0697 & 0.050 \\
\hline 311.0 & 312.0 & 4.7780 & 5.4000 & 0.6220 & 69.3106 & 4.0711 & 4.0635 & 0.186 \\
\hline 312.0 & 313.0 & 5.4000 & 6.0510 & 0.6510 & 69.3105 & 4.0764 & 4.0573 & 0.467 \\
\hline 313.0 & 314.0 & 6.0510 & 6.7090 & 0.6580 & 69.3105 & 4.0458 & 4.0511 & -0.132 \\
\hline 314.0 & 315.0 & 6.7090 & 7.3680 & 0.6590 & 69.3105 & 4.0528 & 4.0449 & 0.194 \\
\hline 315.0 & 316.0 & 7.3680 & 8.0620 & 0.6940 & 69.3105 & 4.0549 & 4.0387 & 0.400 \\
\hline 316.0 & 317.0 & 8.0620 & 8.7550 & 0.6930 & 69.3105 & 4.0452 & 4.0324 & 0.317 \\
\hline 317.0 & 318.0 & 8.7550 & 9.4830 & 0.7280 & 69.3105 & 4.0215 & 4.0260 & -0.113 \\
\hline 318.0 & 319.0 & 9.4830 & 10.2120 & 0.7290 & 69.3105 & 4.0106 & 4.0197 & -0.227 \\
\hline 319.0 & 320.0 & 10.2120 & 10.9540 & 0.7420 & 69.3105 & 4.0343 & 4.0133 & 0.520 \\
\hline 320.0 & 321.0 & 10.9540 & 11.7140 & 0.7600 & 69.3105 & 4.0259 & 4.0069 & 0.471 \\
\hline 321.0 & 322.0 & 11.7140 & 12.4790 & 0.7650 & 69.3104 & 3.9974 & 4.0005 & -0.079 \\
\hline 322.0 & 323.0 & 12.4790 & 13.2680 & 0.7890 & 69.3104 & 4.0170 & 3.9941 & 0.570 \\
\hline 323.0 & 324.0 & 13.2680 & 14.0640 & 0.7960 & 69.3104 & 3.9954 & 3.9877 & 0.194 \\
\hline 324.0 & 325.0 & 14.0640 & 14.8760 & 0.8120 & 69.3104 & 3.9923 & 3.9812 & 0.278 \\
\hline 325.0 & 326.0 & 14.8760 & 15.7000 & 0.8240 & 69.3104 & 3.9822 & 3.9747 & 0.187 \\
\hline 326.0 & 327.0 & 15.7000 & 16.5330 & 0.8330 & 69.3104 & 3.9842 & 3.9683 & 0.400 \\
\hline 327.0 & 328.0 & 16.5330 & 17.3890 & 0.8560 & 69.3104 & 3.9851 & 3.9618 & 0.586 \\
\hline 329.0 & 330.0 & 18.2480 & 19.1220 & 0.8740 & 69.3104 & 3.9776 & 3.9488 & 0.725 \\
\hline 304.0 & 305.0 & 0.9870 & 1.4380 & 0.4510 & 69.3106 & 4.1274 & 4.1053 & 0.536 \\
\hline 305.0 & 306.0 & 1.4380 & 1.9300 & 0.4920 & 69.3106 & 4.1017 & 4.0995 & 0.053 \\
\hline 307.0 & 308.0 & 2.4550 & 3.0040 & 0.5490 & 69.3106 & 4.0892 & 4.0878 & 0.035 \\
\hline 308.0 & 309.0 & 3.0040 & 3.5860 & 0.5820 & 69.3106 & 4.0802 & 4.0818 & -0.038 \\
\hline 309.0 & 310.0 & 3.5860 & 4.1730 & 0.5870 & 69.3106 & 4.0740 & 4.0757 & -0.042 \\
\hline 310.0 & 311.0 & 4.1730 & 4.7790 & 0.6060 & 69.3106 & 4.0721 & 4.0697 & 0.060 \\
\hline 311.0 & 312.0 & 4.7790 & 5.4030 & 0.6240 & 69.3106 & 4.0755 & 4.0635 & 0.294 \\
\hline 312.0 & 313.0 & 5.4030 & 6.0440 & 0.6410 & 69.3105 & 4.0799 & 4.0574 & 0.552 \\
\hline
\end{tabular}


Table 1. Measurements of specific heat capacity at constant volume $c_{V}$ for $\mathrm{H}_{2} \mathrm{O}: T$, temperature (ITS-90); $p$, pressure; $m$, sample mass; $c_{V, \text { exp }}$, experimental heat capacity; $c_{V \text {,calc }}$, heat capacity calculated with the equation of state of Ref. [7]; $D=100$ $\left(c_{V, \exp }-c_{V, \text { calc }}\right) / c_{V, \exp }$-Continued

\begin{tabular}{|c|c|c|c|c|c|c|c|c|}
\hline $\begin{array}{r}T_{1} \\
(\mathrm{~K})\end{array}$ & $\begin{array}{r}T_{2} \\
(\mathrm{~K})\end{array}$ & $\begin{array}{c}p_{1} \\
(\mathrm{MPa})\end{array}$ & $\begin{array}{c}p_{2} \\
(\mathrm{MPa})\end{array}$ & $\begin{array}{c}\Delta p \\
(\mathrm{MPa})\end{array}$ & $\begin{array}{l}m \\
(\mathrm{~g})\end{array}$ & \multicolumn{2}{|c|}{$\left(\mathrm{kJ} \cdot \mathrm{kg}^{-1} \cdot \mathrm{K}^{-1}\right)$} & $\begin{array}{c}D \\
(\%)\end{array}$ \\
\hline 313.0 & 314.0 & 6.0440 & 6.6960 & 0.6520 & 69.3105 & 4.0594 & 4.0512 & 0.203 \\
\hline 314.0 & 315.0 & 6.6960 & 7.3630 & 0.6670 & 69.3105 & 4.0498 & 4.0450 & 0.120 \\
\hline 315.0 & 316.0 & 7.3630 & 8.0630 & 0.7000 & 69.3105 & 4.0565 & 4.0387 & 0.440 \\
\hline 316.0 & 317.0 & 8.0630 & 8.7530 & 0.6900 & 69.3105 & 4.0452 & 4.0324 & 0.317 \\
\hline 317.0 & 318.0 & 8.7530 & 9.4840 & 0.7310 & 69.3105 & 4.0248 & 4.0260 & -0.031 \\
\hline 318.0 & 319.0 & 9.4840 & 10.2140 & 0.7300 & 69.3105 & 4.0222 & 4.0197 & 0.062 \\
\hline 319.0 & 320.0 & 10.2140 & 10.9570 & 0.7430 & 69.3105 & 4.0240 & 4.0133 & 0.265 \\
\hline 320.0 & 321.0 & 10.9570 & 11.7130 & 0.7560 & 69.3105 & 4.0093 & 4.0069 & 0.059 \\
\hline 321.0 & 322.0 & 11.7130 & 12.4860 & 0.7730 & 69.3104 & 4.0137 & 4.0005 & 0.328 \\
\hline 322.0 & 323.0 & 12.4860 & 13.2700 & 0.7840 & 69.3104 & 4.0227 & 3.9941 & 0.711 \\
\hline 323.0 & 324.0 & 13.2700 & 14.0660 & 0.7960 & 69.3104 & 3.9945 & 3.9877 & 0.171 \\
\hline 324.0 & 325.0 & 14.0660 & 14.8750 & 0.8090 & 69.3104 & 3.9862 & 3.9812 & 0.125 \\
\hline 325.0 & 326.0 & 14.8750 & 15.7010 & 0.8260 & 69.3104 & 3.9825 & 3.9747 & 0.195 \\
\hline 327.0 & 328.0 & 16.5370 & 17.3850 & 0.8480 & 69.3104 & 3.9605 & 3.9618 & -0.032 \\
\hline 328.0 & 329.0 & 17.3850 & 18.2460 & 0.8610 & 69.3104 & 3.9444 & 3.9553 & -0.276 \\
\hline 304.0 & 305.0 & 1.0000 & 1.4490 & 0.4490 & 69.3106 & 4.1384 & 4.1052 & 0.802 \\
\hline 305.0 & 306.0 & 1.4490 & 1.9420 & 0.4930 & 69.3106 & 4.1188 & 4.0995 & 0.469 \\
\hline 306.0 & 307.0 & 1.9420 & 2.4640 & 0.5220 & 69.3106 & 4.1187 & 4.0937 & 0.608 \\
\hline 307.0 & 308.0 & 2.4640 & 3.0100 & 0.5460 & 69.3106 & 4.1002 & 4.0877 & 0.304 \\
\hline 308.0 & 309.0 & 3.0100 & 3.5770 & 0.5670 & 69.3106 & 4.0950 & 4.0818 & 0.323 \\
\hline 309.0 & 310.0 & 3.5770 & 4.1630 & 0.5860 & 69.3106 & 4.0895 & 4.0758 & 0.336 \\
\hline 310.0 & 311.0 & 4.1630 & 4.7760 & 0.6130 & 69.3106 & 4.0890 & 4.0697 & 0.473 \\
\hline 311.0 & 312.0 & 4.7760 & 5.4200 & 0.6440 & 69.3106 & 4.0734 & 4.0635 & 0.243 \\
\hline 312.0 & 313.0 & 5.4200 & 6.0610 & 0.6410 & 69.3105 & 4.0577 & 4.0573 & 0.009 \\
\hline 313.0 & 314.0 & 6.0610 & 6.7120 & 0.6510 & 69.3105 & 4.0454 & 4.0511 & -0.142 \\
\hline 314.0 & 315.0 & 6.7120 & 7.3650 & 0.6530 & 69.3105 & 4.0224 & 4.0450 & -0.561 \\
\hline 316.0 & 317.0 & 8.0670 & 8.7720 & 0.7050 & 69.3105 & 4.0414 & 4.0323 & 0.224 \\
\hline 317.0 & 318.0 & 8.7720 & 9.4850 & 0.7130 & 69.3105 & 4.0310 & 4.0260 & 0.123 \\
\hline 318.0 & 319.0 & 9.4850 & 10.2120 & 0.7270 & 69.3105 & 4.0396 & 4.0197 & 0.493 \\
\hline 319.0 & 320.0 & 10.2120 & 10.9610 & 0.7490 & 69.3105 & 4.0168 & 4.0133 & 0.087 \\
\hline 321.0 & 322.0 & 11.7180 & 12.4860 & 0.7680 & 69.3104 & 3.9925 & 4.0005 & -0.201 \\
\hline 322.0 & 323.0 & 12.4860 & 13.2740 & 0.7880 & 69.3104 & 4.0119 & 3.9941 & 0.444 \\
\hline 323.0 & 324.0 & 13.2740 & 14.0690 & 0.7950 & 69.3104 & 3.9863 & 3.9877 & -0.034 \\
\hline 324.0 & 325.0 & 14.0690 & 14.8790 & 0.8100 & 69.3104 & 3.9806 & 3.9812 & -0.015 \\
\hline 325.0 & 326.0 & 14.8790 & 15.7040 & 0.8250 & 69.3104 & 3.9959 & 3.9747 & 0.530 \\
\hline 326.0 & 327.0 & 15.7040 & 16.5420 & 0.8380 & 69.3104 & 3.9936 & 3.9682 & 0.635 \\
\hline 327.0 & 328.0 & 16.5420 & 17.3900 & 0.8480 & 69.3104 & 3.9586 & 3.9618 & -0.080 \\
\hline 328.0 & 329.0 & 17.3900 & 18.2530 & 0.8630 & 69.3104 & 3.9525 & 3.9553 & -0.070 \\
\hline 323.0 & 324.0 & 2.3540 & 3.1390 & 0.7850 & 68.9132 & 3.9967 & 4.0144 & -0.442 \\
\hline 325.0 & 326.0 & 3.9350 & 4.7440 & 0.8090 & 68.9131 & 3.9873 & 4.0008 & -0.339 \\
\hline 326.0 & 327.0 & 4.7440 & 5.5660 & 0.8220 & 68.9131 & 4.0043 & 3.9940 & 0.257 \\
\hline 327.0 & 328.0 & 5.5660 & 6.4000 & 0.8340 & 68.9131 & 3.9799 & 3.9872 & -0.183 \\
\hline 328.0 & 329.0 & 6.4000 & 7.2470 & 0.8470 & 68.9131 & 3.9739 & 3.9804 & -0.163 \\
\hline 329.0 & 330.0 & 7.2470 & 8.1020 & 0.8550 & 68.9131 & 3.9953 & 3.9736 & 0.544 \\
\hline 331.0 & 332.0 & 8.9740 & 9.8560 & 0.8820 & 68.9131 & 3.9763 & 3.9600 & 0.411 \\
\hline 332.0 & 333.0 & 9.8560 & 10.7490 & 0.8930 & 68.9130 & 3.9474 & 3.9531 & -0.145 \\
\hline 333.0 & 334.0 & 10.7490 & 11.6520 & 0.9030 & 68.9130 & 3.9317 & 3.9463 & -0.372 \\
\hline 334.0 & 335.0 & 11.6520 & 12.5690 & 0.9170 & 68.9130 & 3.9424 & 3.9395 & 0.073 \\
\hline 335.0 & 336.0 & 12.5690 & 13.4960 & 0.9270 & 68.9130 & 3.9282 & 3.9327 & -0.115 \\
\hline 336.0 & 337.0 & 13.4960 & 14.4350 & 0.9390 & 68.9130 & 3.9193 & 3.9259 & -0.169 \\
\hline 337.0 & 338.0 & 14.4350 & 15.3820 & 0.9470 & 68.9130 & 3.9059 & 3.9192 & -0.339 \\
\hline 338.0 & 339.0 & 15.3820 & 16.3420 & 0.9600 & 68.9130 & 3.9062 & 3.9124 & -0.158 \\
\hline 339.0 & 340.0 & 16.3420 & 17.3110 & 0.9690 & 68.9130 & 3.8996 & 3.9056 & -0.154 \\
\hline 341.0 & 342.0 & 18.2880 & 19.2790 & 0.9910 & 68.9129 & 3.8973 & 3.8921 & 0.133 \\
\hline 326.0 & 327.0 & 4.7430 & 5.5650 & 0.8220 & 68.9131 & 3.9846 & 3.9940 & -0.236 \\
\hline 327.0 & 328.0 & 5.5650 & 6.3990 & 0.8340 & 68.9131 & 3.9744 & 3.9872 & -0.322 \\
\hline
\end{tabular}


Table 1. Measurements of specific heat capacity at constant volume $c_{V}$ for $\mathrm{H}_{2} \mathrm{O}: T$, temperature (ITS-90); $p$, pressure; $m$, sample mass; $c_{V, \text { exp }}$, experimental heat capacity; $c_{V, \text { calc }}$, heat capacity calculated with the equation of state of Ref. [7]; $D=100$ $\left(c_{V, \exp }-c_{V, \text { calc }}\right) / c_{V, \exp }$-Continued

\begin{tabular}{|c|c|c|c|c|c|c|c|c|}
\hline $\begin{array}{r}T_{1} \\
(\mathrm{~K})\end{array}$ & $\begin{array}{r}T_{2} \\
(\mathrm{~K})\end{array}$ & $\begin{array}{c}p_{1} \\
(\mathrm{MPa})\end{array}$ & $\begin{array}{c}p_{2} \\
(\mathrm{MPa})\end{array}$ & $\begin{array}{c}\Delta p \\
(\mathrm{MPa})\end{array}$ & $\begin{array}{l}m \\
(\mathrm{~g})\end{array}$ & \multicolumn{2}{|c|}{$\begin{array}{c}c_{V, \exp } c_{V, \text { calc }} \\
\left(\mathrm{kJ} \cdot \mathrm{kg}^{-1} \cdot \mathrm{K}^{-1}\right)\end{array}$} & $\begin{array}{c}D \\
(\%)\end{array}$ \\
\hline 328.0 & 329.0 & 6.3990 & 7.2460 & 0.8470 & 68.9131 & 3.9835 & 3.9804 & 0.078 \\
\hline 329.0 & 330.0 & 7.2460 & 8.1030 & 0.8570 & 68.9131 & 3.9781 & 3.9736 & 0.114 \\
\hline 330.0 & 331.0 & 8.1030 & 8.9750 & 0.8720 & 68.9131 & 3.9510 & 3.9668 & -0.399 \\
\hline 331.0 & 332.0 & 8.9750 & 9.8560 & 0.8810 & 68.9131 & 3.9488 & 3.9600 & -0.282 \\
\hline 333.0 & 334.0 & 10.7510 & 11.6530 & 0.9020 & 68.9130 & 3.9276 & 3.9463 & -0.477 \\
\hline 334.0 & 335.0 & 11.6530 & 12.5700 & 0.9170 & 68.9130 & 3.9409 & 3.9395 & 0.035 \\
\hline 335.0 & 336.0 & 12.5700 & 13.4970 & 0.9270 & 68.9130 & 3.9282 & 3.9327 & -0.115 \\
\hline 337.0 & 338.0 & 14.4320 & 15.3830 & 0.9510 & 68.9130 & 3.8941 & 3.9192 & -0.643 \\
\hline 338.0 & 339.0 & 15.3830 & 16.3390 & 0.9560 & 68.9130 & 3.9130 & 3.9124 & 0.016 \\
\hline 339.0 & 340.0 & 16.3390 & 17.3110 & 0.9720 & 68.9130 & 3.9013 & 3.9056 & -0.110 \\
\hline 340.0 & 341.0 & 17.3110 & 18.2880 & 0.9770 & 68.9130 & 3.8830 & 3.8989 & -0.408 \\
\hline 341.0 & 342.0 & 18.2880 & 19.2780 & 0.9900 & 68.9129 & 3.8904 & 3.8921 & -0.044 \\
\hline 326.0 & 327.0 & 4.7340 & 5.5570 & 0.8230 & 68.9131 & 4.0266 & 3.9940 & 0.809 \\
\hline 328.0 & 329.0 & 6.3900 & 7.2390 & 0.8490 & 68.9131 & 3.9915 & 3.9804 & 0.278 \\
\hline 329.0 & 330.0 & 7.2390 & 8.0960 & 0.8570 & 68.9131 & 3.9892 & 3.9736 & 0.391 \\
\hline 330.0 & 331.0 & 8.0960 & 8.9680 & 0.8720 & 68.9131 & 3.9426 & 3.9668 & -0.613 \\
\hline 331.0 & 332.0 & 8.9680 & 9.8490 & 0.8810 & 68.9131 & 3.9557 & 3.9600 & -0.108 \\
\hline 332.0 & 333.0 & 9.8490 & 10.7420 & 0.8930 & 68.9131 & 3.9565 & 3.9532 & 0.084 \\
\hline 333.0 & 334.0 & 10.7420 & 11.6480 & 0.9060 & 68.9130 & 3.9613 & 3.9463 & 0.377 \\
\hline 334.0 & 335.0 & 11.6480 & 12.5660 & 0.9180 & 68.9130 & 3.9286 & 3.9395 & -0.278 \\
\hline 335.0 & 336.0 & 12.5660 & 13.4930 & 0.9270 & 68.9130 & 3.9402 & 3.9327 & 0.189 \\
\hline 336.0 & 337.0 & 13.4930 & 14.4300 & 0.9370 & 68.9130 & 3.9164 & 3.9259 & -0.244 \\
\hline 338.0 & 339.0 & 15.3810 & 16.3380 & 0.9570 & 68.9130 & 3.8844 & 3.9124 & -0.720 \\
\hline 340.0 & 341.0 & 17.3070 & 18.2850 & 0.9780 & 68.9130 & 3.8838 & 3.8989 & -0.388 \\
\hline 344.0 & 345.0 & 4.1610 & 5.1580 & 0.9970 & 68.2821 & 3.8903 & 3.9052 & -0.384 \\
\hline 346.0 & 347.0 & 6.1670 & 7.1830 & 1.0160 & 68.2821 & 3.8884 & 3.8910 & -0.068 \\
\hline 348.0 & 349.0 & 8.2150 & 9.2530 & 1.0380 & 68.2821 & 3.8908 & 3.8769 & 0.357 \\
\hline 349.0 & 350.0 & 9.2530 & 10.2990 & 1.0460 & 68.2821 & 3.8877 & 3.8699 & 0.458 \\
\hline 350.0 & 351.0 & 10.2990 & 11.3590 & 1.0600 & 68.2820 & 3.8768 & 3.8629 & 0.359 \\
\hline 351.0 & 352.0 & 11.3590 & 12.4220 & 1.0630 & 68.2820 & 3.8547 & 3.8559 & -0.031 \\
\hline 352.0 & 353.0 & 12.4220 & 13.4980 & 1.0760 & 68.2820 & 3.8535 & 3.8490 & 0.118 \\
\hline 353.0 & 354.0 & 13.4980 & 14.5800 & 1.0820 & 68.2820 & 3.8580 & 3.8420 & 0.414 \\
\hline 355.0 & 356.0 & 15.6690 & 16.7680 & 1.0990 & 68.2820 & 3.8481 & 3.8283 & 0.516 \\
\hline 356.0 & 357.0 & 16.7680 & 17.8720 & 1.1040 & 68.2820 & 3.8183 & 3.8214 & -0.081 \\
\hline 357.0 & 358.0 & 17.8720 & 18.9860 & 1.1140 & 68.2820 & 3.8053 & 3.8146 & -0.244 \\
\hline 343.0 & 344.0 & 3.1830 & 4.1660 & 0.9830 & 68.2821 & 3.9207 & 3.9123 & 0.213 \\
\hline 344.0 & 345.0 & 4.1660 & 5.1600 & 0.9940 & 68.2821 & 3.8980 & 3.9052 & -0.185 \\
\hline 345.0 & 346.0 & 5.1600 & 6.1690 & 1.0090 & 68.2821 & 3.9127 & 3.8981 & 0.373 \\
\hline 346.0 & 347.0 & 6.1690 & 7.1860 & 1.0170 & 68.2821 & 3.8969 & 3.8910 & 0.151 \\
\hline 347.0 & 348.0 & 7.1860 & 8.2150 & 1.0290 & 68.2821 & 3.8825 & 3.8840 & -0.038 \\
\hline 348.0 & 349.0 & 8.2150 & 9.2490 & 1.0340 & 68.2821 & 3.9049 & 3.8769 & 0.716 \\
\hline 349.0 & 350.0 & 9.2490 & 10.2980 & 1.0490 & 68.2821 & 3.8755 & 3.8699 & 0.145 \\
\hline 350.0 & 351.0 & 10.2980 & 11.3520 & 1.0540 & 68.2820 & 3.8674 & 3.8629 & 0.116 \\
\hline 351.0 & 352.0 & 11.3520 & 12.4200 & 1.0680 & 68.2820 & 3.8762 & 3.8559 & 0.523 \\
\hline 352.0 & 353.0 & 12.4200 & 13.4910 & 1.0710 & 68.2820 & 3.8483 & 3.8490 & -0.017 \\
\hline 353.0 & 354.0 & 13.4910 & 14.5750 & 1.0840 & 68.2820 & 3.8484 & 3.8420 & 0.165 \\
\hline 355.0 & 356.0 & 15.6680 & 16.7650 & 1.0970 & 68.2820 & 3.8405 & 3.8283 & 0.319 \\
\hline 356.0 & 357.0 & 16.7650 & 17.8710 & 1.1060 & 68.2820 & 3.8116 & 3.8214 & -0.257 \\
\hline 357.0 & 358.0 & 17.8710 & 18.9860 & 1.1150 & 68.2820 & 3.8232 & 3.8146 & 0.225 \\
\hline 346.0 & 347.0 & 6.1610 & 7.1800 & 1.0190 & 68.2821 & 3.9018 & 3.8910 & 0.276 \\
\hline 347.0 & 348.0 & 7.1800 & 8.2100 & 1.0300 & 68.2821 & 3.8829 & 3.8840 & -0.028 \\
\hline 348.0 & 349.0 & 8.2100 & 9.2480 & 1.0380 & 68.2821 & 3.9060 & 3.8769 & 0.744 \\
\hline 349.0 & 350.0 & 9.2480 & 10.2960 & 1.0480 & 68.2821 & 3.8682 & 3.8699 & -0.044 \\
\hline 350.0 & 351.0 & 10.2960 & 11.3530 & 1.0570 & 68.2820 & 3.8858 & 3.8629 & 0.589 \\
\hline 351.0 & 352.0 & 11.3530 & 12.4200 & 1.0670 & 68.2820 & 3.8622 & 3.8559 & 0.163 \\
\hline 353.0 & 354.0 & 13.4900 & 14.5730 & 1.0830 & 68.2820 & 3.8594 & 3.8420 & 0.450 \\
\hline
\end{tabular}


Table 1. Measurements of specific heat capacity at constant volume $c_{V}$ for $\mathrm{H}_{2} \mathrm{O}: T$, temperature (ITS-90); $p$, pressure; $m$, sample mass; $c_{V, \text { exp }}$, experimental heat capacity; $c_{V \text {,calc }}$, heat capacity calculated with the equation of state of Ref. [7]; $D=100$ $\left(c_{V, \exp }-c_{V, \text { calc }}\right) / c_{V, \exp }$-Continued

\begin{tabular}{|c|c|c|c|c|c|c|c|c|}
\hline $\begin{array}{r}T_{1} \\
(\mathrm{~K})\end{array}$ & $\begin{array}{r}T_{2} \\
(\mathrm{~K})\end{array}$ & $\begin{array}{c}p_{1} \\
(\mathrm{MPa})\end{array}$ & $\begin{array}{c}p_{2} \\
(\mathrm{MPa})\end{array}$ & $\begin{array}{c}\Delta p \\
(\mathrm{MPa})\end{array}$ & $\begin{array}{l}m \\
(\mathrm{~g})\end{array}$ & \multicolumn{2}{|c|}{$\left(\mathrm{kJ} \cdot \mathrm{kg}^{-1} \cdot \mathrm{K}^{-1}\right)$} & $\begin{array}{c}D \\
(\%)\end{array}$ \\
\hline 354.0 & 355.0 & 14.5730 & 15.6650 & 1.0920 & 68.2820 & 3.8591 & 3.8351 & 0.621 \\
\hline 355.0 & 356.0 & 15.6650 & 16.7630 & 1.0980 & 68.2820 & 3.8387 & 3.8283 & 0.272 \\
\hline 356.0 & 357.0 & 16.7630 & 17.8690 & 1.1060 & 68.2820 & 3.8265 & 3.8214 & 0.133 \\
\hline 357.0 & 358.0 & 17.8690 & 18.9840 & 1.1150 & 68.2820 & 3.8220 & 3.8146 & 0.194 \\
\hline 362.0 & 363.0 & 2.6960 & 3.8200 & 1.1240 & 67.4994 & 3.8018 & 3.8148 & -0.343 \\
\hline 364.0 & 365.0 & 4.9580 & 6.1100 & 1.1520 & 67.4993 & 3.8034 & 3.8007 & 0.071 \\
\hline 365.0 & 366.0 & 6.1100 & 7.2640 & 1.1540 & 67.4993 & 3.7902 & 3.7937 & -0.092 \\
\hline 366.0 & 367.0 & 7.2640 & 8.4340 & 1.1700 & 67.4993 & 3.7752 & 3.7867 & -0.305 \\
\hline 368.0 & 369.0 & 9.6050 & 10.7850 & 1.1800 & 67.4993 & 3.7638 & 3.7729 & -0.241 \\
\hline 370.0 & 371.0 & 11.9680 & 13.1630 & 1.1950 & 67.4992 & 3.7645 & 3.7592 & 0.142 \\
\hline 371.0 & 372.0 & 13.1630 & 14.3610 & 1.1980 & 67.4992 & 3.7418 & 3.7524 & -0.283 \\
\hline 372.0 & 373.0 & 14.3610 & 15.5620 & 1.2010 & 67.4992 & 3.7242 & 3.7456 & -0.575 \\
\hline 373.0 & 374.0 & 15.5620 & 16.7710 & 1.2090 & 67.4992 & 3.7163 & 3.7389 & -0.608 \\
\hline 374.0 & 375.0 & 16.7710 & 17.9910 & 1.2200 & 67.4992 & 3.7062 & 3.7322 & -0.702 \\
\hline 362.0 & 363.0 & 2.6960 & 3.8200 & 1.1240 & 67.4994 & 3.8052 & 3.8148 & -0.253 \\
\hline 363.0 & 364.0 & 3.8200 & 4.9580 & 1.1380 & 67.4993 & 3.7887 & 3.8078 & -0.503 \\
\hline 364.0 & 365.0 & 4.9580 & 6.1080 & 1.1500 & 67.4993 & 3.7802 & 3.8007 & -0.542 \\
\hline 366.0 & 367.0 & 7.2660 & 8.4320 & 1.1660 & 67.4993 & 3.7713 & 3.7867 & -0.409 \\
\hline 367.0 & 368.0 & 8.4320 & 9.6030 & 1.1710 & 67.4993 & 3.7539 & 3.7798 & -0.689 \\
\hline 368.0 & 369.0 & 9.6030 & 10.7840 & 1.1810 & 67.4993 & 3.7683 & 3.7729 & -0.121 \\
\hline 369.0 & 370.0 & 10.7840 & 11.9680 & 1.1840 & 67.4993 & 3.7470 & 3.7660 & -0.507 \\
\hline 370.0 & 371.0 & 11.9680 & 13.1600 & 1.1920 & 67.4992 & 3.7477 & 3.7592 & -0.306 \\
\hline 371.0 & 372.0 & 13.1600 & 14.3580 & 1.1980 & 67.4992 & 3.7537 & 3.7524 & 0.035 \\
\hline 372.0 & 373.0 & 14.3580 & 15.5660 & 1.2080 & 67.4992 & 3.7337 & 3.7456 & -0.319 \\
\hline 373.0 & 374.0 & 15.5660 & 16.7710 & 1.2050 & 67.4992 & 3.7241 & 3.7389 & -0.398 \\
\hline 374.0 & 375.0 & 16.7710 & 17.9900 & 1.2190 & 67.4992 & 3.6977 & 3.7322 & -0.934 \\
\hline 362.0 & 363.0 & 2.6850 & 3.8120 & 1.1270 & 67.4994 & 3.8043 & 3.8148 & -0.277 \\
\hline 363.0 & 364.0 & 3.8120 & 4.9510 & 1.1390 & 67.4994 & 3.8016 & 3.8078 & -0.162 \\
\hline 364.0 & 365.0 & 4.9510 & 6.1020 & 1.1510 & 67.4993 & 3.7799 & 3.8007 & -0.551 \\
\hline 366.0 & 367.0 & 7.2600 & 8.4260 & 1.1660 & 67.4993 & 3.7689 & 3.7867 & -0.473 \\
\hline 367.0 & 368.0 & 8.4260 & 9.5970 & 1.1710 & 67.4993 & 3.7665 & 3.7798 & -0.353 \\
\hline 368.0 & 369.0 & 9.5970 & 10.7770 & 1.1800 & 67.4993 & 3.7753 & 3.7729 & 0.064 \\
\hline 369.0 & 370.0 & 10.7770 & 11.9620 & 1.1850 & 67.4993 & 3.7303 & 3.7660 & -0.957 \\
\hline 371.0 & 372.0 & 13.1560 & 14.3540 & 1.1980 & 67.4992 & 3.7279 & 3.7524 & -0.657 \\
\hline 372.0 & 373.0 & 14.3540 & 15.5580 & 1.2040 & 67.4992 & 3.7290 & 3.7456 & -0.446 \\
\hline 373.0 & 374.0 & 15.5580 & 16.7670 & 1.2090 & 67.4992 & 3.7244 & 3.7389 & -0.390 \\
\hline 374.0 & 375.0 & 16.7670 & 17.9870 & 1.2200 & 67.4992 & 3.7094 & 3.7322 & -0.615 \\
\hline 384.0 & 385.0 & 5.2340 & 6.4890 & 1.2550 & 66.5677 & 3.6618 & 3.6986 & -1.005 \\
\hline 385.0 & 386.0 & 6.4890 & 7.7490 & 1.2600 & 66.5676 & 3.6715 & 3.6919 & -0.557 \\
\hline 386.0 & 387.0 & 7.7490 & 9.0170 & 1.2680 & 66.5676 & 3.6587 & 3.6853 & -0.728 \\
\hline 387.0 & 388.0 & 9.0170 & 10.2910 & 1.2740 & 66.5676 & 3.6481 & 3.6787 & -0.840 \\
\hline 388.0 & 389.0 & 10.2910 & 11.5690 & 1.2780 & 66.5676 & 3.6596 & 3.6722 & -0.344 \\
\hline 389.0 & 390.0 & 11.5690 & 12.8490 & 1.2800 & 66.5676 & 3.6275 & 3.6657 & -1.053 \\
\hline 391.0 & 392.0 & 14.1330 & 15.4230 & 1.2900 & 66.5676 & 3.6148 & 3.6528 & -1.052 \\
\hline 392.0 & 393.0 & 15.4230 & 16.7150 & 1.2920 & 66.5675 & 3.6268 & 3.6465 & -0.542 \\
\hline 393.0 & 394.0 & 16.7150 & 18.0130 & 1.2980 & 66.5675 & 3.6123 & 3.6401 & -0.771 \\
\hline 394.0 & 395.0 & 18.0130 & 19.3160 & 1.3030 & 66.5675 & 3.6042 & 3.6339 & -0.823 \\
\hline 383.0 & 384.0 & 3.9870 & 5.2350 & 1.2480 & 66.5677 & 3.7024 & 3.7053 & -0.079 \\
\hline 384.0 & 385.0 & 5.2350 & 6.4880 & 1.2530 & 66.5677 & 3.6589 & 3.6986 & -1.085 \\
\hline 385.0 & 386.0 & 6.4880 & 7.7480 & 1.2600 & 66.5676 & 3.6645 & 3.6919 & -0.749 \\
\hline 386.0 & 387.0 & 7.7480 & 9.0160 & 1.2680 & 66.5676 & 3.6332 & 3.6853 & -1.435 \\
\hline 387.0 & 388.0 & 9.0160 & 10.2910 & 1.2750 & 66.5676 & 3.6334 & 3.6787 & -1.248 \\
\hline 388.0 & 389.0 & 10.2910 & 11.5650 & 1.2740 & 66.5676 & 3.6238 & 3.6722 & -1.336 \\
\hline 389.0 & 390.0 & 11.5650 & 12.8480 & 1.2830 & 66.5676 & 3.6245 & 3.6657 & -1.137 \\
\hline 390.0 & 391.0 & 12.8480 & 14.1330 & 1.2850 & 66.5676 & 3.6130 & 3.6593 & -1.280 \\
\hline 391.0 & 392.0 & 14.1330 & 15.4250 & 1.2920 & 66.5676 & 3.6068 & 3.6528 & -1.276 \\
\hline
\end{tabular}


Table 1. Measurements of specific heat capacity at constant volume $c_{V}$ for $\mathrm{H}_{2} \mathrm{O}: T$, temperature (ITS-90); $p$, pressure; $m$, sample mass; $c_{V, \text { exp }}$, experimental heat capacity; $c_{V \text {,calc }}$, heat capacity calculated with the equation of state of Ref. [7]; $D=100$ $\left(c_{V, \exp }-c_{V, \text { calc }}\right) / c_{V, \exp }$-Continued

\begin{tabular}{|c|c|c|c|c|c|c|c|c|}
\hline $\begin{array}{r}T_{1} \\
(\mathrm{~K})\end{array}$ & $\begin{array}{r}T_{2} \\
(\mathrm{~K})\end{array}$ & $\begin{array}{c}p_{1} \\
(\mathrm{MPa})\end{array}$ & $\begin{array}{c}p_{2} \\
(\mathrm{MPa})\end{array}$ & $\begin{array}{c}\Delta p \\
(\mathrm{MPa})\end{array}$ & $\begin{array}{l}m \\
(\mathrm{~g})\end{array}$ & \multicolumn{2}{|c|}{$\left(\mathrm{kJ} \cdot \mathrm{kg}^{-1} \cdot \mathrm{K}^{-1}\right)$} & $\begin{array}{c}D \\
(\%)\end{array}$ \\
\hline 392.0 & 393.0 & 15.4250 & 16.7150 & 1.2900 & 66.5675 & 3.6203 & 3.6465 & -0.723 \\
\hline 394.0 & 395.0 & 18.0150 & 19.3170 & 1.3020 & 66.5675 & 3.5999 & 3.6339 & -0.943 \\
\hline 383.0 & 384.0 & 3.9890 & 5.2380 & 1.2490 & 66.5677 & 3.6882 & 3.7053 & -0.464 \\
\hline 384.0 & 385.0 & 5.2380 & 6.4930 & 1.2550 & 66.5677 & 3.6722 & 3.6986 & -0.719 \\
\hline 386.0 & 387.0 & 7.7530 & 9.0210 & 1.2680 & 66.5676 & 3.6588 & 3.6853 & -0.725 \\
\hline 387.0 & 388.0 & 9.0210 & 10.2920 & 1.2710 & 66.5676 & 3.6504 & 3.6787 & -0.776 \\
\hline 389.0 & 390.0 & 11.5710 & 12.8530 & 1.2820 & 66.5676 & 3.6378 & 3.6657 & -0.767 \\
\hline 390.0 & 391.0 & 12.8530 & 14.1370 & 1.2840 & 66.5676 & 3.6414 & 3.6592 & -0.490 \\
\hline 391.0 & 392.0 & 14.1370 & 15.4290 & 1.2920 & 66.5675 & 3.6240 & 3.6528 & -0.796 \\
\hline 392.0 & 393.0 & 15.4290 & 16.7200 & 1.2910 & 66.5675 & 3.6287 & 3.6465 & -0.490 \\
\hline 393.0 & 394.0 & 16.7200 & 18.0200 & 1.3000 & 66.5675 & 3.6134 & 3.6401 & -0.740 \\
\hline 394.0 & 395.0 & 18.0200 & 19.3230 & 1.3030 & 66.5675 & 3.6076 & 3.6339 & -0.728 \\
\hline 383.0 & 384.0 & 3.9960 & 5.2430 & 1.2470 & 66.5677 & 3.6990 & 3.7053 & -0.170 \\
\hline 384.0 & 385.0 & 5.2430 & 6.5010 & 1.2580 & 66.5677 & 3.6896 & 3.6986 & -0.244 \\
\hline 386.0 & 387.0 & 7.7620 & 9.0270 & 1.2650 & 66.5676 & 3.6654 & 3.6853 & -0.543 \\
\hline 387.0 & 388.0 & 9.0270 & 10.3030 & 1.2760 & 66.5676 & 3.6604 & 3.6787 & -0.501 \\
\hline 388.0 & 389.0 & 10.3030 & 11.5780 & 1.2750 & 66.5676 & 3.6642 & 3.6722 & -0.218 \\
\hline 389.0 & 390.0 & 11.5780 & 12.8570 & 1.2790 & 66.5676 & 3.6567 & 3.6657 & -0.246 \\
\hline 390.0 & 391.0 & 12.8570 & 14.1420 & 1.2850 & 66.5676 & 3.6623 & 3.6592 & 0.084 \\
\hline 392.0 & 393.0 & 15.4240 & 16.7180 & 1.2940 & 66.5675 & 3.6453 & 3.6465 & -0.032 \\
\hline 393.0 & 394.0 & 16.7180 & 18.0200 & 1.3020 & 66.5675 & 3.6056 & 3.6401 & -0.958 \\
\hline 394.0 & 395.0 & 18.0200 & 19.3220 & 1.3020 & 66.5675 & 3.6158 & 3.6339 & -0.499 \\
\hline 404.0 & 405.0 & 5.5430 & 6.8680 & 1.3250 & 65.5150 & 3.5958 & 3.6007 & -0.137 \\
\hline 405.0 & 406.0 & 6.8680 & 8.1970 & 1.3290 & 65.5150 & 3.5899 & 3.5945 & -0.130 \\
\hline 406.0 & 407.0 & 8.1970 & 9.5280 & 1.3310 & 65.5150 & 3.5823 & 3.5884 & -0.170 \\
\hline 407.0 & 408.0 & 9.5280 & 10.8640 & 1.3360 & 65.5150 & 3.5661 & 3.5823 & -0.455 \\
\hline 408.0 & 409.0 & 10.8640 & 12.1990 & 1.3350 & 65.5149 & 3.5626 & 3.5763 & -0.383 \\
\hline 409.0 & 410.0 & 12.1990 & 13.5440 & 1.3450 & 65.5149 & 3.5626 & 3.5702 & -0.214 \\
\hline 410.0 & 411.0 & 13.5440 & 14.8900 & 1.3460 & 65.5149 & 3.5595 & 3.5643 & -0.134 \\
\hline 411.0 & 412.0 & 14.8900 & 16.2370 & 1.3470 & 65.5149 & 3.5489 & 3.5583 & -0.266 \\
\hline 412.0 & 413.0 & 16.2370 & 17.5870 & 1.3500 & 65.5149 & 3.5457 & 3.5525 & -0.191 \\
\hline 403.0 & 404.0 & 4.2280 & 5.5470 & 1.3190 & 65.5150 & 3.5929 & 3.6070 & -0.391 \\
\hline 404.0 & 405.0 & 5.5470 & 6.8680 & 1.3210 & 65.5150 & 3.6009 & 3.6007 & 0.005 \\
\hline 405.0 & 406.0 & 6.8680 & 8.1970 & 1.3290 & 65.5150 & 3.6066 & 3.5945 & 0.334 \\
\hline 406.0 & 407.0 & 8.1970 & 9.5310 & 1.3340 & 65.5150 & 3.5984 & 3.5884 & 0.278 \\
\hline 407.0 & 408.0 & 9.5310 & 10.8680 & 1.3370 & 65.5150 & 3.5794 & 3.5823 & -0.081 \\
\hline 409.0 & 410.0 & 12.2040 & 13.5500 & 1.3460 & 65.5149 & 3.5541 & 3.5702 & -0.454 \\
\hline 410.0 & 411.0 & 13.5500 & 14.8930 & 1.3430 & 65.5149 & 3.5339 & 3.5643 & -0.859 \\
\hline 413.0 & 414.0 & 17.5900 & 18.9450 & 1.3550 & 65.5149 & 3.5334 & 3.5466 & -0.374 \\
\hline 403.0 & 404.0 & 4.2220 & 5.5450 & 1.3230 & 65.5150 & 3.5855 & 3.6070 & -0.599 \\
\hline 404.0 & 405.0 & 5.5450 & 6.8680 & 1.3230 & 65.5150 & 3.5884 & 3.6007 & -0.344 \\
\hline 405.0 & 406.0 & 6.8680 & 8.1990 & 1.3310 & 65.5150 & 3.5846 & 3.5945 & -0.278 \\
\hline 406.0 & 407.0 & 8.1990 & 9.5320 & 1.3330 & 65.5150 & 3.5723 & 3.5884 & -0.451 \\
\hline 407.0 & 408.0 & 9.5320 & 10.8720 & 1.3400 & 65.5150 & 3.5776 & 3.5823 & -0.131 \\
\hline 408.0 & 409.0 & 10.8720 & 12.2100 & 1.3380 & 65.5149 & 3.5519 & 3.5762 & -0.685 \\
\hline 410.0 & 411.0 & 13.5550 & 14.8970 & 1.3420 & 65.5149 & 3.5281 & 3.5643 & -1.025 \\
\hline 412.0 & 413.0 & 16.2490 & 17.5970 & 1.3480 & 65.5149 & 3.5189 & 3.5524 & -0.953 \\
\hline 413.0 & 414.0 & 17.5970 & 18.9520 & 1.3550 & 65.5149 & 3.5284 & 3.5466 & -0.516 \\
\hline
\end{tabular}




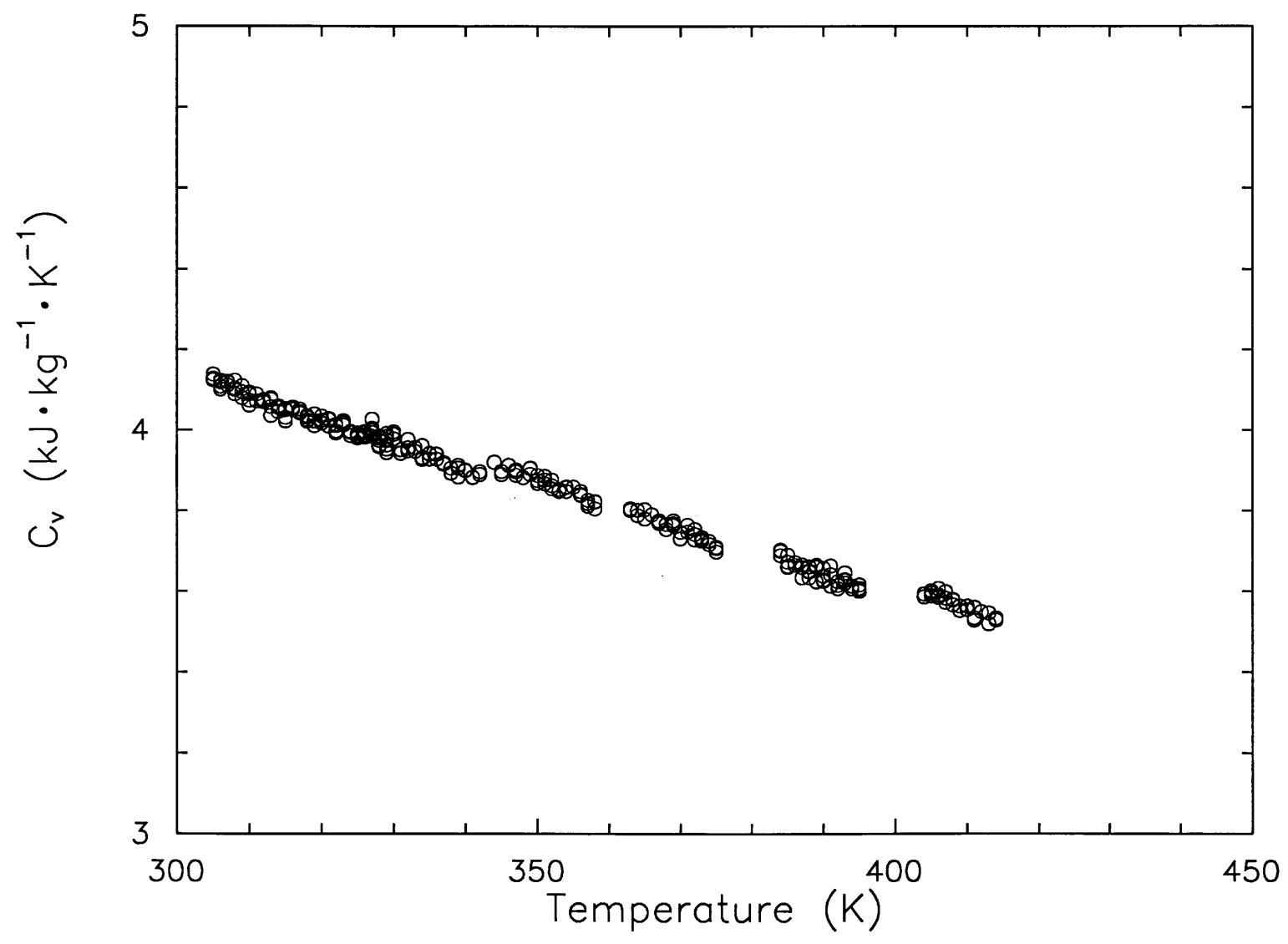

Fig. 3. Measured specific heat capacity at constant volume $c_{V}$ for $\mathrm{H}_{2} \mathrm{O}$.

calculations with predictive models, this table gives more significant figures than would be normally justified. Experimental temperatures ( $T$, ITS-90), pressures $(p)$, and masses $(m)$ are presented alongside the heat capacity $\left(c_{V}\right)$ data. These state values were used to establish the volume of the sample bomb. This quantity was calculated from $m / V_{\text {calc }}(T, p)$, where $V_{\text {calc }}$ is calculated with the equation of state in the NBS/NRC Steam Tables of Haar, Gallagher, and Kell [6]. The calculated volume $\left(V_{\mathrm{b}}\right)$, as a function of both temperature $(T)$ and pressure $(p)$, was fitted to the equation,

$$
V_{\mathrm{b}}=\left[V_{\mathrm{r}}+c_{1}(T-273.15 \mathrm{~K})\right]\left[1+c_{2} p\right]
$$

where $V_{\mathrm{r}}=69.464 \mathrm{~cm}^{3}, c_{1}=3.2 \times 10^{-3} \mathrm{~cm}^{3} \cdot \mathrm{K}^{-1}$, and $c_{2}=1.36 \times 10^{-4} \mathrm{MPa}^{-1}$. Figure 4 shows the deviations of experimental densities from those calculated with the recently adopted international standard formulation by Pru $\beta$ and Wagner [7]. Since these experimental densities were determined from the mass of water found in Table 1 and the bomb volume calculated with Eq. (4), this comparison is intended to test how well Eq. (4) represents the sample bomb volume as a function of $T$ and $p$.
Deviations from the equation of state of Ref. [7] were not greater than $0.1 \%$ and gave a root-mean-square fractional deviation of $0.05 \%$. Since we have found that densities calculated with Ref. [6] differ by less than $0.006 \%$ from those calculated with Ref. [7] in the range of $T$ and $p$ of this work, we could justify the use of either of these formulations for our comparisons.

Comparisons of the $c_{V}$ measurements were made with published values. Figures 5 and 6 shows comparisons with the calculations based on the Pru $\beta$ and Wagner formulation [7] at temperatures from $300 \mathrm{~K}$ to $420 \mathrm{~K}$. The deviations of $c_{V}$ from this study shown in Fig. 5 did not exceed $\pm 1 \%$ and gave a root-mean-square fractional deviation of $0.48 \%$. Figure 5 shows good agreement of this work with the published $c_{V}$ data of Amirkhanov et al. [8], which have an uncertainty of approximately $3 \%$. Figure 5 illustrates that deviations of the present data are distributed uniformly above and below the baseline representing the calculation of Pru $\beta$ and Wagner. The same comment applies to the published data of Ref. [8], except that they fall within a $\pm 3 \%$ band. Since no other $c_{V}$ data were found in the temperature range of this study, we decided to make 


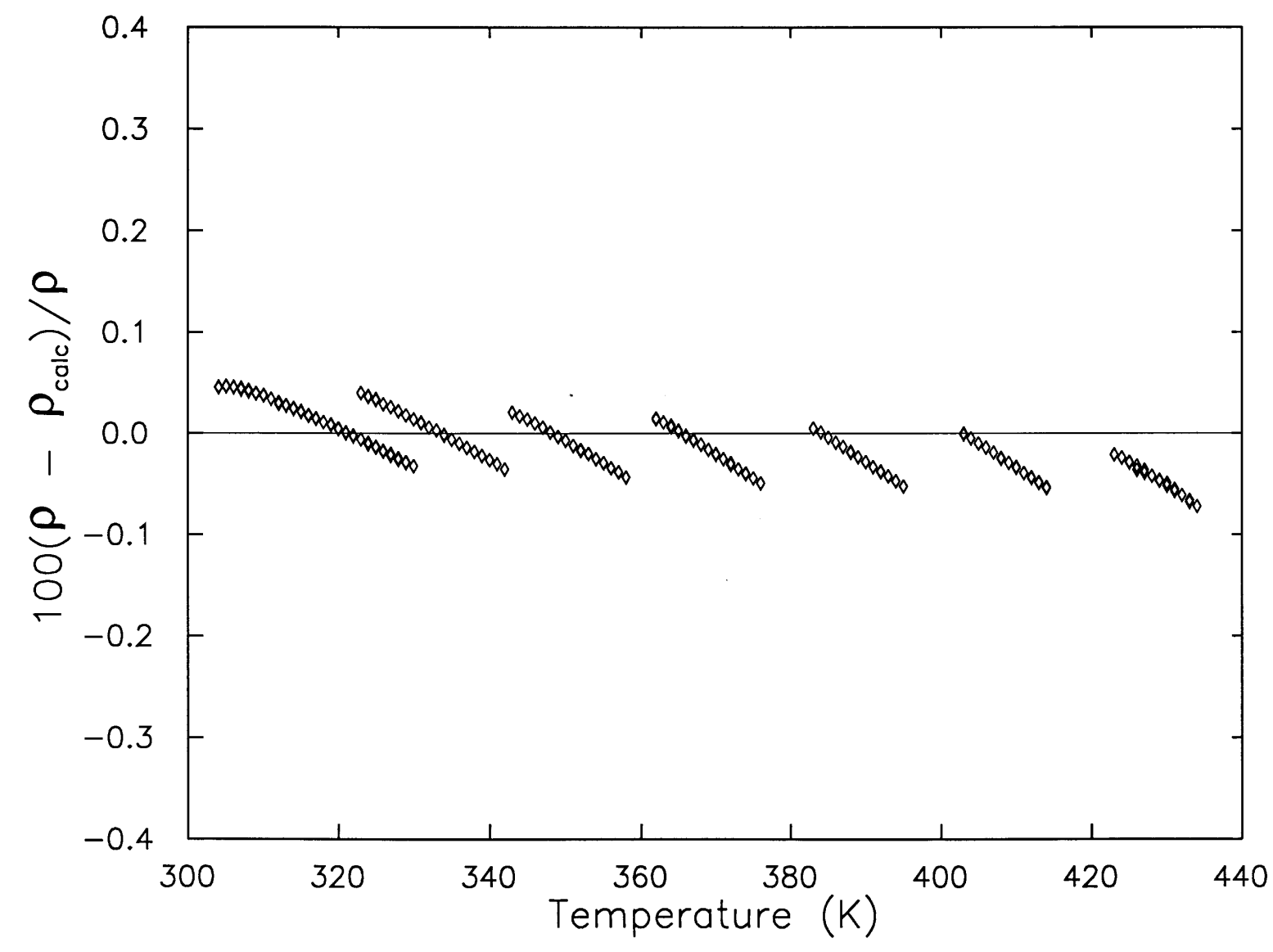

Fig. 4. Deviations of $\mathrm{H}_{2} \mathrm{O}$ densities as determined in this work $(\diamond)$ from densities calculated with the equation of state of Pru $\beta$ and Wagner [7] (baseline at deviation $=0.0$ ).

indirect comparisons with published specific heat capacity at constant pressure $c_{p}$ data from Sirota and Mal'tsev [9], which have an uncertainty of approximately $1 \%$. Figure 6 shows the deviations of the $c_{p}$ data from calculations made with the Pru $\beta$ and Wagner formulation. All deviations of the Ref. [9] data are within the claimed uncertainty of the published data, and fall in $\mathrm{a} \pm 0.2 \%$ band. Based on both direct and indirect comparisons, we conclude that the present results are in very good agreement with published $c_{V}$ and $c_{p}$ data.

\section{Assessment of Uncertainties}

Uncertainty in $c_{V}$ arises from several sources. Primarily, the accuracy of this method is limited by the uncertainty involved in the temperature rise measurement and the change-of-volume work adjustment [3]. In the following discussion, we use a definition for the expanded uncertainty which is two times the standard uncertainty (i.e., a coverage factor $k=2$ and thus a 2-standard-deviation estimate).
Different sources of uncertainty, including calibration of the platinum resistance thermometer, radiation to or from the thermometer head, and drift of the ice point resistance, contribute to an expanded uncertainty of $3 \times 10^{-2} \mathrm{~K}$ for the absolute temperature measurement. Uncertainty in the temperature rise measurement, however, also depends on the reproducibility of temperature measurements. The temperatures assigned to the beginning $\left(T_{1}\right)$ and to the end $\left(T_{2}\right)$ of a heating interval are determined from a linear fit of temperature with elapsed time, near the integer degree. The experimental ramp rate is approximately $+4 \times 10^{-2} \mathrm{~K} \cdot \mathrm{min}^{-1}$. This procedure leads to an uncertainty of $5 \times 10^{-4} \mathrm{~K}$ for the interpolated temperatures $T_{1}$ and $T_{2}$, leading to values of $7 \times 10^{-4} \mathrm{~K}$ for the uncertainty of the temperature rise, $\Delta T=T_{2}-T_{1}$. For a typical experimental value of $\Delta T=1 \mathrm{~K}$, this corresponds to a relative uncertainty of $0.07 \%$.

The uncertainty of the change-of-volume work adjustment influences primarily the single-phase values since two-phase experiments are performed over a small pressure range. For water, the ratio of change-of-volume 


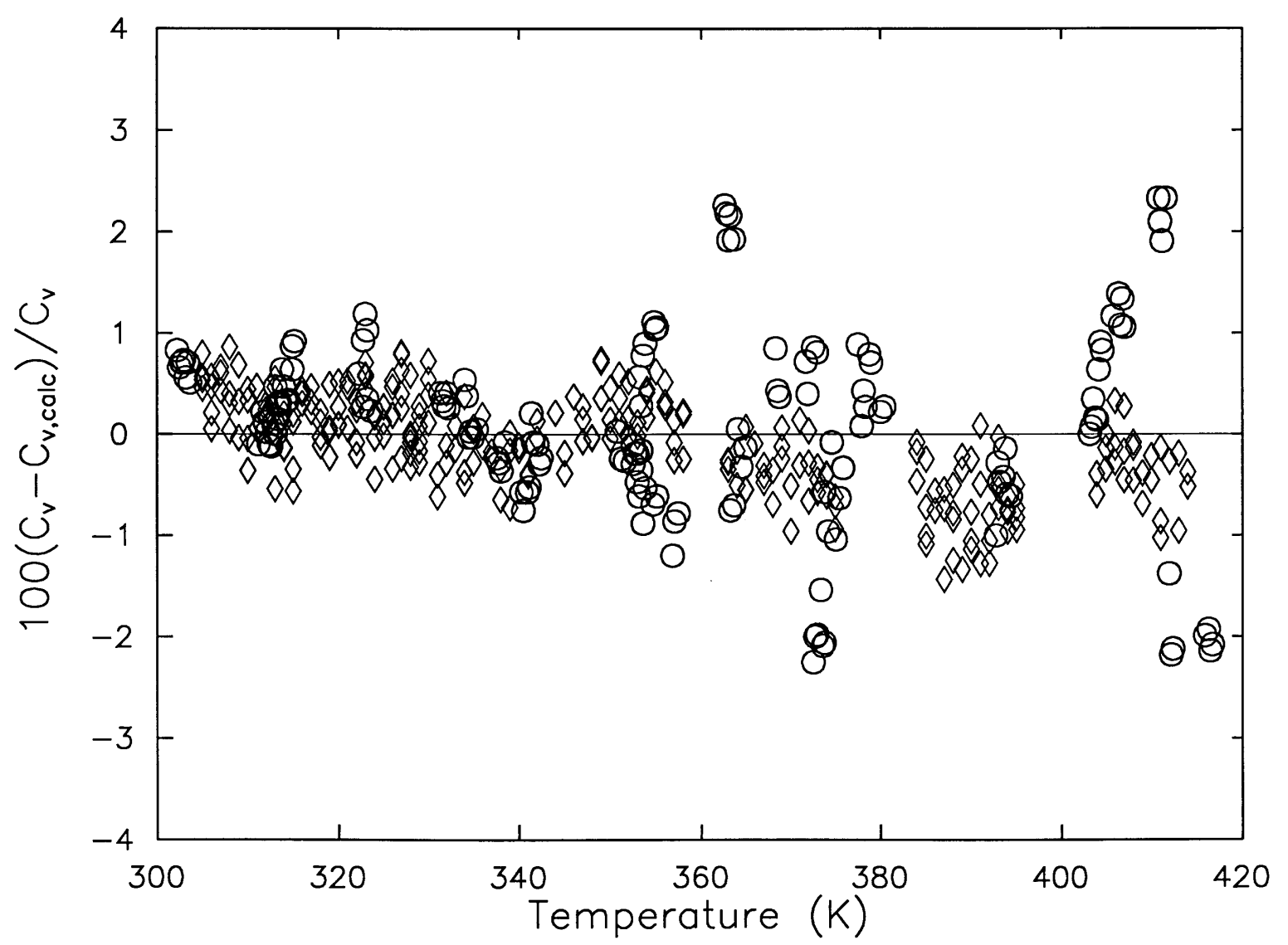

Fig. 5. Deviation of measured $\mathrm{H}_{2} \mathrm{O}$ specific heat capacities at constant volume $c_{V}$ of this work $(\diamond)$ and of Ref. [8] (O) from heat capacities calculated with the equation of state of Pru $\beta$ and Wagner [7] (baseline at deviation $=0.0$ ).

work to total applied heat is as large as 0.04 for the lowest density isochore. Estimated relative uncertainties of $2 \%$ in the change-of-volume work are due to both the deviation of the calculated pressure derivatives and the uncertainty of the volume change. This leads to a relative uncertainty in $c_{V}$ of $0.08 \%$.

The energy applied to the calorimeter is the integral of the product of voltage and current from the initial to the final heating time. Voltage and current are measured 80 times during a heating interval of $1 \mathrm{~K}$. The measurements of the electrical quantities have a relative uncertainty of $0.02 \%$. However, we must account for the effect of radiation heat losses or gains which occur when a spurious lag of the controller leads to a small temperature difference of about $10^{-2} \mathrm{~K}$ between bomb and radiation shield. Since heat transfer by radiation is proportional to $T_{1}^{4}-T_{2}^{4} \approx 4 T^{3} \Delta T$, we would expect radiation losses to substantially increase with the bomb temperature, and the losses may be different from the sample and from the reference bombs. Therefore, the uncertainty in the applied heat $\Delta Q$ is evaluated to be 0.5 $\mathrm{J} \cdot \mathrm{K}^{-1}$.
The energy difference $\Delta Q_{0}$ applied to the empty calorimeter has been measured in repeated experiments and fitted to a function of temperature. Its uncertainty is less than $0.5 \mathrm{~J} \cdot \mathrm{K}^{-1}$. Its influence on the uncertainty of the heat capacity is relatively small, because the ratio of the total heat $\Delta Q$ to the heat applied to the empty calorimeter $\Delta Q_{0}$ ranges from 100 to 600 . The mass of each sample was determined within $0.01 \%$ by differential weighings before and after trapping the sample. The density calculated from this mass and the bomb volume has a relative uncertainty of approximately $0.2 \%$. For pressures, the uncertainty of the gauge of $7 \mathrm{kPa}$ is added to the cross term for the pressure derivative in the change-of-volume work adjustment. However, neither the uncertainty of $p$ nor $\rho$ contributes appreciably to the combined uncertainty for the measured heat capacity. The relative uncertainty of $c_{V}$ is determined to be $0.3 \%$, by combining the various sources of experimental uncertainty using a root-sum-of-squares formula. 


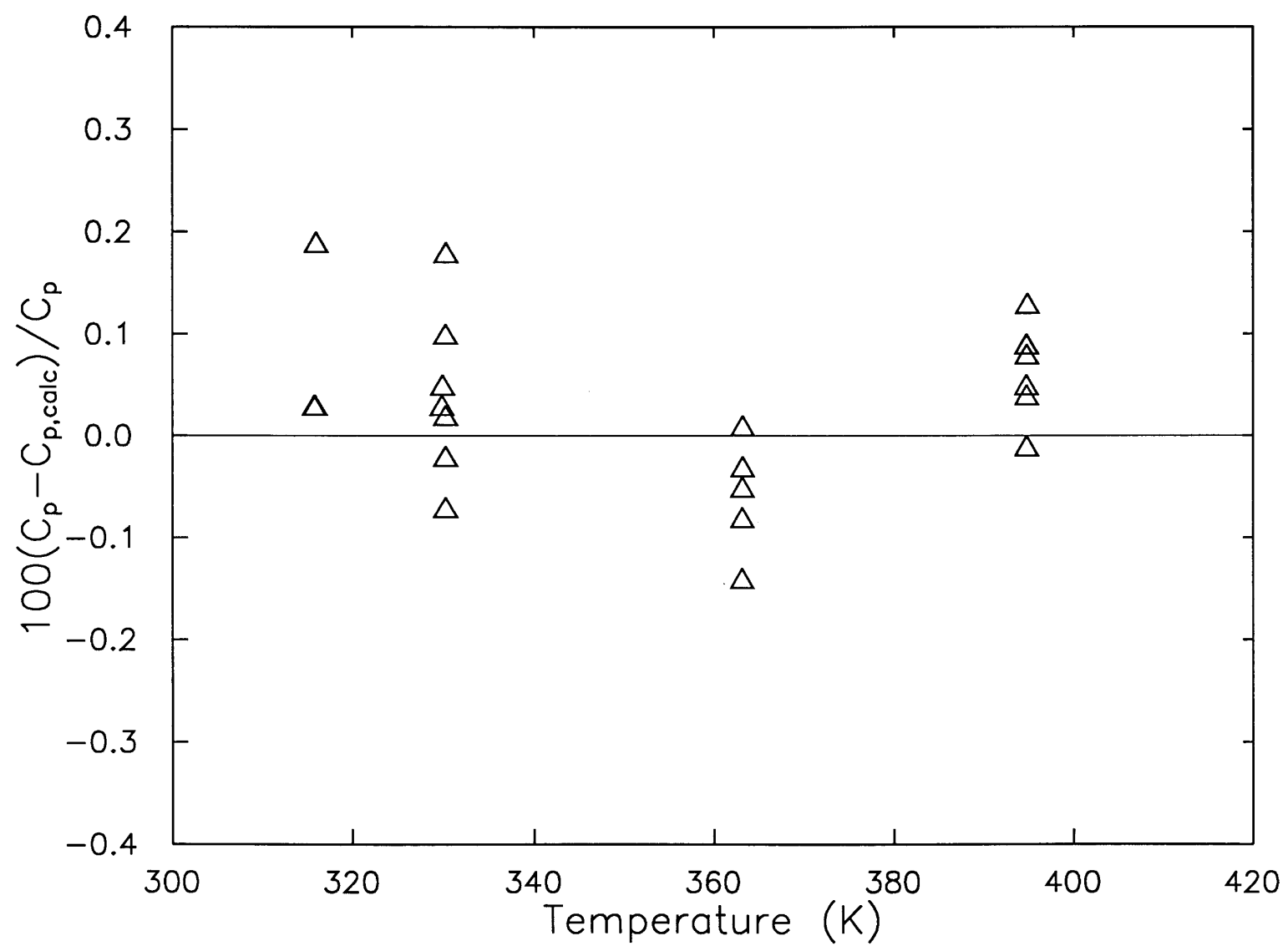

Fig. 6. Deviation of measured $\mathrm{H}_{2} \mathrm{O}$ specific heat capacities at constant pressure $c_{p}$ of Ref. [9] $(\diamond)$ from heat capacities calculated with the equation of state of Pruß and Wagner [7] (baseline at deviation =0.0).

\section{Acknowledgments}

This work was supported by the United States Department of Energy, Division of Engineering and Geosciences, Office of Basic Energy Sciences. RJD and JCB acknowledge the financial support of NIST Professional Research Experience Program Fellowships. We gratefully acknowledge the dedication to excellence of our team of scientific instrument makers: Tim Waldorf, Bob Gomez, and Mike Rybowiak.

\section{References}

[1] J. P. McCullough and D. W. Scott, eds., Experimental Thermodynamics Volume I. Calorimetry of Non-reacting Systems, Butterworths, London (1967).

[2] R. D. Goodwin, J. Res. Natl. Bur. Stand. (U.S.) 65C, 231-243 (1961).

[3] J. W. Magee, J. Res. Natl. Inst. Stand. Technol. 96, 725-740 (1991).

[4] J. P. Joule, Mem. Proc. Manchester Lit. Phil. Soc. 2, 559 (1845).
[5] L. Pfaundler, Sitzber. Akad. Wiss. Wien, Math. Naturw. Kl. 59, 145 (1869).

[6] L. Haar, J. S. Gallagher, and G. S. Kell, NBS/NRC Steam Tables, Hemisphere, Washington, DC (1984).

[7] A. Pru $\beta$ and W. Wagner, Release on the IAPWS Formulation 1995 for the Thermodynamic Properties of Ordinary Water Substance for General and Scientific Use, Fredericia, Denmark, September 1996.

[8] Kh. I. Amirkhanov, G. V. Stepanov, and B. G. Alibekov, Isochoric Heat Capacity of Water and Steam, M. P. Vukalovich, ed., (Akad. Nauk SSSR, Dagestanskii Filial, 1969). English Translation Amerind Publ. Co., New Delhi (1974).

[9] A. M. Sirota and B. K. Mal'tsev, Teploenegetika 6, 7 (1959).

About the authors: Joseph Magee is a chemical engineer with the Physical and Chemical Properties Division of the NIST Chemical Science and Technology Laboratory. Renee Deal and John Blanco have each been granted a degree in engineering by the University of Colorado, and are now pursuing careers in industry. The National Institute of Standards and Technology is an agency of the Technology Administration, U.S. Department of Commerce. 